\title{
LINEABILITY AND SPACEABILITY FOR THE WEAK FORM OF PEANO'S THEOREM AND VECTOR-VALUED SEQUENCE SPACES
}

\author{
CLEON S. BARROSO, GERALDO BOTELHO, VINÍCIUS V. FÁVARO, \\ AND DANIEL PELLEGRINO
}

(Communicated by Michael T. Lacey)

\begin{abstract}
Two new applications of a technique for spaceability are given in this paper. For the first time this technique is used in the investigation of the algebraic genericity property of the weak form of Peano's theorem on the existence of solutions of the ODE $u^{\prime}=f(u)$ on $c_{0}$. The space of all continuous vector fields $f$ on $c_{0}$ is proved to contain a closed $\mathfrak{c}$-dimensional subspace formed by fields $f$ for which, except for the null field, the weak form of Peano's theorem fails to be true. The second application generalizes known results on the existence of closed $\mathfrak{c}$-dimensional subspaces inside certain subsets of $\ell_{p}(X)$-spaces, $0<p<\infty$, to the existence of closed subspaces of maximal dimension inside such subsets.
\end{abstract}

\section{INTRODUCTION}

The notions of lineability and spaceability, as well as their applications, have been heavily studied lately by many authors in different settings; see, e.g., 1, 2, 4, 7, 8, 12, 16 and the references therein. The basic task in the field consists in finding linear structures, as large as possible, inside nonempty sets with certain properties. Usually, given a cardinal number $\mu$ and a (remarkable) subset $A$ of a topological vector space $E$, one wishes to show that $A \cup\{0\}$ contains a $\mu$-dimensional subspace of $E$. According to the usual terminology, $A$ is said to be:

- $\mu$-lineable if $A \cup\{0\}$ contains a $\mu$-dimensional subspace of $E$;

- $\mu$-spaceable if $A \cup\{0\}$ contains a closed $\mu$-dimensional subspace of $E$;

- maximal-spaceable if it is $\mu$-spaceable with $\mu=\operatorname{dim} E$.

A standard methodology to verify such properties consists of making a convenient manipulation of a single element of $A$ in order to define an injective linear operator $T: X \longrightarrow E$, where $X$ is an infinite dimensional Banach space, such that $T(X) \subseteq A \cup\{0\}$. In this case $A$ is $\operatorname{dim} X$-lineable, and if $\overline{T(X)} \subseteq A \cup\{0\}$, then $A$ is $\operatorname{dim} X$-spaceable. The starting element of $A$ can be called the mother vector. The purpose of this paper is to discuss two new applications of the mother vector

Received by the editors June 2, 2011 and, in revised form, September 24, 2011.

2010 Mathematics Subject Classification. Primary 15A03, 46B45, 34A12.

The first author was supported by CNPq Grant 307210/2009-0.

The second author was supported by CNPq Grant 306981/2008-4.

The third author was supported by FAPEMIG Grant CEX-APQ-00208-09.

The fourth author was supported by CNPq Grant 301237/2009-3 and CAPES-NF. 
technique. First, it is explored in a situation for which it was never applied before (cf. Section 2). Thereafter, the mother vector technique is used to obtain maximalspaceability in a more general framework than that where spaceability was obtained in [4] (cf. Section 3). Next we briefly describe the results we prove by means of the mother vector technique.

Throughout this paper, $\mathfrak{c}$ will denote the cardinality of the continuum. Given a Banach space $X$, we denote by $\mathscr{K}(X)$ the set of all continuous vector fields $f: X \longrightarrow X$ for which the weak form of Peano's theorem, concerning the existence of local solutions of

$$
u^{\prime}(t)=f(u(t)),
$$

fails to be true. For more details on this current line of research and a historical account, we refer the reader to the recent contribution of Hájek and Johanis [1] and the references therein. In Section 2 the mother vector technique is used to prove that $\mathscr{K}\left(c_{0}\right)$ is spaceable in the space $C\left(c_{0}\right)$ of all continuous vector fields on $c_{0}$ endowed with the topology of uniform convergence on bounded subsets. We call this type of property the algebraic genericity of differential equations in $X$. The motivation comes from studies on the generic property of differential equations in Banach spaces (see [13). Our approach to prove this result is based on Dieudonné's construction of vector fields on $c_{0}$ failing the classical Peano's theorem (cf. 6]). To the best of our knowledge, this is the first time spaceability is studied in this context.

Our next study concerns maximal-spaceability in the setting of vector-valued sequence spaces. In [4, the mother vector technique was used to prove that if $X$ is a Banach space, then $\ell_{p}(X)-\bigcup_{q<p} \ell_{q}(X), 0<p<\infty$, and $c_{0}(X)-\bigcup_{q>0} \ell_{q}(X)$ are spaceable. In Section 3 we refine the application of the mother vector technique in this context by proving that these sets are actually maximal-spaceable. Furthermore, it is proved that, for $1 \leq p<\infty, \bigcap_{p<q} \ell_{q}(X)-\ell_{p}(X)$ is maximal-spaceable in the Fréchet space $\bigcap_{p<q} \ell_{q}(X)$. These results are obtained as particular cases of spaceability results we prove in the more general realm of $\left(\sum_{n} X_{n}\right)_{p}$-spaces. As far as we know, maximal-spaceability with dimension greater than $\mathfrak{c}$ was obtained before only in [8] for sets of nonmeasurable functions.

\section{The WEAK Form of PEANO'S THEOREM IN $c_{0}$}

Let $X$ be a Banach space and $f: \mathbb{R} \times X \longrightarrow X$ be a continuous vector field on $X$. The weak form of Peano's theorem states that if $X$ is finite-dimensional, then the ODE

$$
u^{\prime}=f(t, u)
$$

has a solution on some open interval $I$ in $\mathbb{R}$. The study of the failure of Peano's theorem in arbitrary infinite-dimensional linear spaces was started by Dieudonné [6] in 1950. He proved the existence of a continuous vector field $f: c_{0} \longrightarrow c_{0}$ such that if $f(t, u):=f(u)$, then the Cauchy-Peano problem associated to (1) has no local solution around the null vector of $\mathbb{R} \times c_{0}$. Subsequently, counterexamples in Hilbert spaces and in nonreflexive Banach spaces were obtained by Yorke [20, Godunov [10] and Cellina [5, respectively. Finally, in 1973 Godunov 9] proved that Peano's theorem holds true in $X$ if and only if $X$ is finite-dimensional. Further negative 
answers were obtained by Astala [3, Shkarin [18, 19, Lobanov 14, and Lobanov and Smolyanov [15] in the setting of locally convex and Fréchet spaces.

Let $C(X)$ denote the linear space of all continuous vector fields on $X$, which we endow with the linear topology of uniform convergence on bounded sets. From the lineability and spaceability point of view, the following question emerges naturally: How large is the set $\mathscr{K}(X)$ of all fields $f$ in $C(X)$ for which (11) has no local solution? Following the historical development of the subject, it is natural to investigate the case $X=c_{0}$ first. In this section we give a major step in the solution of the aforementioned question by proving that the set $\mathscr{K}\left(c_{0}\right) \cup\{0\}$ contains a closed subspace of $C\left(c_{0}\right)$.

Theorem 2.1. The set of continuous vector fields on $c_{0}$ failing the weak form of Peano's theorem is spaceable in $C\left(c_{0}\right)$.

Proof. Let $\left(e_{n}\right)_{n=1}^{\infty}$ be the canonical unit vectors of sequence spaces and define the vector field $f \in C\left(c_{0}\right)$ by

$$
f\left(\sum_{n=1}^{\infty} x_{n} e_{n}\right)=\sum_{n=1}^{\infty}\left(\sqrt{\left|x_{n}\right|}+\frac{1}{n+1}\right) e_{n} .
$$

By [6] it follows that $f \in \mathscr{K}\left(c_{0}\right)$. Split $\mathbb{N}$ into countably many infinite pairwise disjoint subsets $\left(\mathbb{N}_{i}\right)_{i=1}^{\infty}$. For every $i \in \mathbb{N}$, set $\mathbb{N}_{i}=\left\{i_{1}<i_{2}<\ldots\right\}$ and define the spreading function $\mathbb{N}_{i} f: c_{0} \longrightarrow c_{0}$ of $f$ over $\mathbb{N}_{i}$ by

$$
\mathbb{N}_{i} f(x)=\sum_{n=1}^{\infty} f_{i_{n}}(x) e_{i_{n}},
$$

where $f_{n}(x)=\sqrt{\left|x_{n}\right|}+\frac{1}{n+1}$, for all $n \in \mathbb{N}$. Let us see that the map

$$
L: \ell_{1} \longrightarrow C\left(c_{0}\right), L\left(\left(a_{i}\right)_{i=1}^{\infty}\right)=\sum_{i=1}^{\infty} a_{i} \mathbb{N}_{i} f
$$

is well defined. Indeed, given $\left(a_{i}\right)_{i=1}^{\infty} \in \ell_{1}$ and $x, y \in c_{0}$, for every $m \in \mathbb{N}$ we have (the sup norm on $c_{0}$ is simply denoted by $\|\cdot\|$ )

$$
\begin{aligned}
\left\|\sum_{i=1}^{m} a_{i} \mathbb{N}_{i} f(x)-\sum_{i=1}^{m} a_{i} \mathbb{N}_{i} f(y)\right\| & \leq \sum_{i=1}^{m}\left|a_{i}\right| \cdot\left\|\mathbb{N}_{i} f(x)-\mathbb{N}_{i} f(y)\right\| \\
& \leq\|f(x)-f(y)\|\left(\sum_{i=1}^{m}\left|a_{i}\right|\right) .
\end{aligned}
$$

First take $y=0$ and let $m \longrightarrow \infty$ to conclude that $\sum_{i=1}^{\infty} a_{i} \mathbb{N}_{i} f(x) \in c_{0}$. Now, letting $m \longrightarrow \infty$ for arbitrary $x$ and $y$, the continuity of $\sum_{i=1}^{\infty} a_{i} \mathbb{N}_{i} f$ follows from the continuity of $f$. Thus $L\left(\left(a_{i}\right)_{i=1}^{\infty}\right) \in C\left(c_{0}\right)$. Since $L$ is well defined, its linearity and injectivity are clear, so the range space $L\left(\ell_{1}\right)$ is algebraically isomorphic to $\ell_{1}$. We claim that

$$
\overline{L\left(\ell_{1}\right)} \subseteq \mathscr{K}\left(c_{0}\right) \cup\{0\} .
$$

Let $h=\left(h_{i}\right)_{i=1}^{\infty} \in \overline{L\left(\ell_{1}\right)}$ be arbitrary. We may assume that $h \neq 0$, so there is $r \in \mathbb{N}$ such that $h_{r} \neq 0$. Using the decomposition $\mathbb{N}=\bigcup_{j=1}^{\infty} \mathbb{N}_{j}$ there are (unique) $m, s \in \mathbb{N}$ 
such that $e_{m_{s}}=e_{r}$. Let $\left(x_{k}\right)_{k=1}^{\infty}=\left(\left(a_{i}^{k}\right)_{i=1}^{\infty}\right)_{k=1}^{\infty}$ be a sequence in $\ell_{1}$ so that

$$
L\left(x_{k}\right)=\sum_{j=1}^{\infty} a_{j}^{k} \mathbb{N}_{j} \stackrel{k \rightarrow \infty}{\longrightarrow} h \text { in } C\left(c_{0}\right) .
$$

Letting $L_{n}\left(x_{k}\right)$ denote the $n$-th coordinate of $L\left(x_{k}\right)$, for each $N \in \mathbb{N}$ we have that

$$
h_{n}=\lim _{k \rightarrow \infty} L_{n}\left(x_{k}\right)
$$

uniformly in the ball $B_{c_{0}}(N):=\left\{x \in c_{0}:\|x\| \leq N\right\}$. Since $L_{i_{j}}\left(x_{k}\right)=a_{i}^{k} f_{i_{j}}$ for all $i, j \in \mathbb{N}$, it follows that

$$
a_{i}^{k} f_{i_{j}}(x)=L_{i_{j}}\left(x_{k}\right)(x) \stackrel{k \rightarrow \infty}{\longrightarrow} h_{i_{j}}(x)
$$

for each $x \in B_{c_{0}}(N)$. In particular,

$$
a_{m}^{k} f_{m_{j}}(x)=L_{m_{j}}\left(x_{k}\right)(x) \stackrel{k \rightarrow \infty}{\longrightarrow} h_{m_{j}}(x)
$$

for every $j$ and every $x \in B_{c_{0}}(N)$, and making $j=s$ we get

$$
a_{m}^{k} f_{r}(x)=a_{m}^{k} f_{m_{s}}(x) \stackrel{k \rightarrow \infty}{\longrightarrow} h_{m_{s}}(x)=h_{r}(x)
$$

for each $x \in B_{c_{0}}(N)$. Choosing $x_{0} \in c_{0}$ such that $h_{r}\left(x_{0}\right) \neq 0$ and $N_{0} \in \mathbb{N}$ such that $x_{0} \in B_{c_{0}}\left(N_{0}\right)$ it follows that

$$
a_{r}:=\lim _{k \rightarrow \infty} a_{m}^{k}=\frac{h_{r}\left(x_{0}\right)}{f_{r}\left(x_{0}\right)} \neq 0 .
$$

Notice that (4) implies $h_{r}(x)=a_{r} f_{r}(x)$ for all $x \in B_{c_{0}}(N)$. As $N$ is arbitrary, we have $h_{r}(x)=a_{r} f_{r}(x)$ for every $x \in c_{0}$. Since for every $j, k \in \mathbb{N}$ the $m_{j}$-th coordinate of $L\left(x_{k}\right)$ is $a_{m}^{k} f_{m_{j}}$, by (3) we have that $h_{m_{j}}(x)=a_{r} f_{m_{j}}(x)$, for all $j \in \mathbb{N}$.

Now we are ready to prove that $h \in \mathscr{K}\left(c_{0}\right)$. We proceed by contradiction using the ODE approach from [6]. Assume that $u(t)=\left(u_{n}(t)\right)_{n=1}^{\infty}$ is a solution of (10) on some interval $I \subset \mathbb{R}$. Fix any $a \in I$ and write $b=u(a)=\left(b_{i}\right)_{i=1}^{\infty}$. In this case we have

$$
u_{m_{j}}^{\prime}(t)=h_{m_{j}}(u(t))=a_{r} f_{m_{j}}(u(t))=a_{r}\left(\sqrt{\left|u_{m_{j}}(t)\right|}+\frac{1}{m_{j}+1}\right),
$$

and $u_{m_{j}}(a)=b_{m_{j}}$ for all $j \in \mathbb{N}$ and $t \in I$. In summary, each $u_{m_{j}}$ is a solution of the Cauchy problem

$$
u_{m_{j}}^{\prime}(t)=a_{r}\left(\sqrt{\left|u_{m_{j}}(t)\right|}+\frac{1}{m_{j}+1}\right), u_{m_{j}}(a)=b_{m_{j}},
$$

for all $j \in \mathbb{N}$ and all $t \in I$. Let us recall the original argument of Dieudonné [6]: if $\alpha, \beta \in \mathbb{R}$ and $\gamma>0$, then

$$
\int_{\alpha}^{\beta} \frac{d x}{\sqrt{|x|}+\gamma} \leq 2(\sqrt{|\alpha|}+\mid \sqrt{\beta \mid})
$$

Thus if $w^{\prime}(t)=\lambda(\sqrt{|w(t)|}+\gamma)$ with $\gamma, \lambda>0, t \geq t_{0}$ and $w\left(t_{0}\right)=y_{0}$, then

$$
t-t_{0}=\int_{t_{0}}^{t} \frac{w^{\prime}(s) d s}{\lambda(\sqrt{|w(s)|}+\gamma)}=\frac{1}{\lambda} \int_{w\left(t_{0}\right)}^{w(t)} \frac{d x}{\sqrt{|x|}+\gamma} \leq \frac{2}{\lambda}\left(\sqrt{|w(t)|}+\sqrt{\left|w\left(t_{0}\right)\right|}\right) .
$$


In view of (5), if $a_{r}>0$, then

$$
0<\frac{a_{r}(t-a)}{2} \leq \sqrt{\left|u_{m_{j}}(t)\right|}+\sqrt{\left|u_{m_{j}}(a)\right|}
$$

for all $t>a$ and all $j \in \mathbb{N}$. This contradiction (remember that $\left(u_{m_{j}}(t)\right)_{j \in \mathbb{N}} \in c_{0}$ for $t \in I)$ shows that $h \in \mathscr{K}\left(c_{0}\right)$. If $a_{r}<0$, we can define $v_{m_{j}}(t)=-u_{m_{j}}(t)$ for all $t \in I$ and $j \in \mathbb{N}$. Then $v_{m_{j}}$ is a solution of the Cauchy problem

$$
v_{m_{j}}^{\prime}(t)=-a_{r}\left(\sqrt{\left|v_{m_{j}}(t)\right|}+\frac{1}{m_{j}+1}\right), v_{m_{j}}(a)=-b_{m_{j}},
$$

for all $j \in \mathbb{N}$ and all $t \in I$. Now, since $-a_{r}>0$, using exactly the same argument used before we obtain a contradiction.

\section{VeCtor-VALUed SEQUenCE SPACES}

Let $\left(X_{n}\right)_{n=1}^{\infty}$ be a sequence of Banach spaces over $\mathbb{K}=\mathbb{R}$ or $\mathbb{C}$. Given $0<p<\infty$, by $\left(\sum_{n} X_{n}\right)_{p}$ we mean the vector space of all sequences $\left(x_{n}\right)_{n=1}^{\infty}$ such that $x_{n} \in X_{n}$ for every $n$ and

$$
\left\|\left(x_{n}\right)_{n=1}^{\infty}\right\|_{p}:=\left(\sum_{n=1}^{\infty}\left\|x_{n}\right\|_{X_{n}}^{p}\right)^{1 / p}<\infty .
$$

It is well known that $\left(\left(\sum_{n} X_{n}\right)_{p},\|\cdot\|_{p}\right)$ is a Banach $(p$-Banach if $0<p<1)$ space. Making the obvious modification for $p=0$ we get the Banach space $\left(\sum_{n} X_{n}\right)_{0}$ of norm null sequences with the sup norm. In this fashion,

$$
\left(\sum_{n} X_{n}\right)_{p}^{-}:=\bigcup_{0<q<p}\left(\sum_{n} X_{n}\right)_{q}
$$

can be regarded as a subspace of $\left(\sum_{n} X_{n}\right)_{p}$ and $\bigcup_{p>0}\left(\sum_{n} X_{n}\right)_{p}$ can be regarded as a subspace of $\left(\sum_{n} X_{n}\right)_{0}$.

For a Banach space $X$, the usual $X$-valued sequence spaces $\ell_{p}(X)$ and $c_{0}(X)$, as well as their corresponding subspaces $\ell_{p}^{-}(X):=\bigcup_{0<q<p} \ell_{q}(X)$ and $\bigcup_{p>0} \ell_{p}(X)$, are recovered putting $X_{n}=X$ for every $n$. In [4] it is proved that $\ell_{p}(X)-\ell_{p}^{-}(X)$ and $c_{0}(X)-\bigcup_{p>0} \ell_{p}(X)$ are spaceable. In this section we shall prove that these sets are actually maximal-spaceable (cf. Corollary 3.5). This information will be obtained as a particular case of spaceability results in the more general realm of $\left(\sum_{n} X_{n}\right)_{p}$-spaces.

Definition 3.1. Given a Banach space $X$, a family $\left(X_{i}\right)_{i \in I}$ of Banach spaces is said to contain isomorphs of $X$ uniformly if there are $\delta>0$ and a family of isomorphisms into $R_{i}: X \longrightarrow X_{i}$ such that $\max \left\{\left\|R_{i}\right\|,\left\|R_{i}^{-1}\right\|\right\} \leq \delta$ for every $i \in I$. 
Theorem 3.2. Let $\left(X_{n}\right)_{n=1}^{\infty}$ be a sequence of Banach spaces that contains a subsequence containing isomorphs of the infinite-dimensional Banach space $X$ uniformly. Then:

(a) $\left(\sum_{n} X_{n}\right)_{p}-\left(\sum_{n} X_{n}\right)_{p}^{-}$is $\operatorname{dim} X$-spaceable for every $0<p<\infty$.

(b) $\left(\sum_{n} X_{n}\right)_{0}-\bigcup_{p>0}\left(\sum_{n} X_{n}\right)_{p}$ is $\operatorname{dim} X$-spaceable.

Proof. It is plain that we can assume, without loss of generality, that the sequence $\left(X_{n}\right)_{n=1}^{\infty}$ contains isomorphs of $X$ uniformly. So there are $\delta>0$ and isomorphisms into $R_{n}: X \longrightarrow X_{n}$ such that $\left\|R_{n}\right\| \leq \delta$ and $\left\|R_{n}^{-1}\right\| \leq \delta$ for every $n \in \mathbb{N}$.

(a) Let $\xi=\left(\xi_{j}\right)_{j=1}^{\infty} \in \ell_{p}-\bigcup_{0<q<p} \ell_{q}$. Split $\mathbb{N}$ into countably many infinite pairwise disjoint subsets $\left(\mathbb{N}_{i}\right)_{i=1}^{\infty}$. For every $i \in \mathbb{N}$ set $\mathbb{N}_{i}=\left\{i_{1}<i_{2}<\ldots\right\}$ and, denoting by $\left(e_{n}\right)_{n=1}^{\infty}$ the canonical unit vectors of sequence spaces, define

$$
y_{i}=\sum_{j=1}^{\infty} \xi_{j} e_{i_{j}} \in \mathbb{K}^{\mathbb{N}}
$$

Since $\left\|y_{i}\right\|_{r}=\|\xi\|_{r}$ for every $r>0$, we have that $y_{i} \in \ell_{p}-\bigcup_{0<q<p} \ell_{q}$, for every $i$. For $x=\left(x_{n}\right)_{n=1}^{\infty} \in \mathbb{K}^{\mathbb{N}}$ and $w \in X$ we write

$$
x \otimes w:=\left(x_{n} R_{n}(w)\right)_{n=1}^{\infty} \in \prod_{n=1}^{\infty} X_{n} .
$$

It is clear that, for all $w, w_{1}, w_{2} \in X$ and $\lambda \in \mathbb{K}$,

$$
x \otimes\left(w_{1}+w_{2}\right)=x \otimes w_{1}+x \otimes w_{2} \text { and } \lambda(x \otimes w)=(\lambda x) \otimes w=x \otimes(\lambda x),
$$

which justifies the use of the symbol $\otimes$. Define $\tilde{s}=1$ if $p \geq 1$ and $\tilde{s}=p$ if $0<p<1$. Let $\left(w_{j}\right)_{j=1}^{\infty} \in \ell_{\tilde{s}}(X)$ be given. As $y_{j} \otimes w_{j} \in \prod_{n=1}^{\infty} X_{n}$ for every $j \in \mathbb{N}$, and

$$
\begin{aligned}
\left\|y_{j} \otimes w_{j}\right\|_{p} & =\left(\sum_{k=1}^{\infty}\left|\xi_{k}\right|^{p} \cdot\left\|R_{j_{k}}\left(w_{j}\right)\right\|_{X_{j_{k}}}^{p}\right)^{\frac{1}{p}} \leq\left(\sum_{k=1}^{\infty}\left|\xi_{k}\right|^{p} \cdot\left\|R_{j_{k}}\right\|^{p} \cdot\left\|w_{j}\right\|_{X}^{p}\right)^{\frac{1}{p}} \\
& \leq \delta\left\|w_{j}\right\|_{X} \cdot\|\xi\|_{p}<\infty,
\end{aligned}
$$

each $y_{j} \otimes w_{j} \in\left(\sum_{n} X_{n}\right)_{p}$. Moreover,

$$
\sum_{j=1}^{\infty}\left\|y_{j} \otimes w_{j}\right\|_{p}^{\tilde{s}} \leq \sum_{j=1}^{\infty}\left(\delta\left\|w_{j}\right\|_{X} \cdot\|\xi\|_{p}\right)^{\tilde{s}}=\delta^{\tilde{s}}\|\xi\|_{p}^{\tilde{s}} \cdot\left\|\left(w_{j}\right)_{j=1}^{\infty}\right\|_{\tilde{s}}^{\tilde{s}}<\infty .
$$

Thus $\sum_{j=1}^{\infty}\left\|y_{j} \otimes w_{j}\right\|_{p}<\infty$ if $p \geq 1$ and $\sum_{j=1}^{\infty}\left\|y_{j} \otimes w_{j}\right\|_{p}^{p}<\infty$ if $0<p<1$. Hence the series $\sum_{j=1}^{\infty} y_{j} \otimes w_{j}$ converges in $\left(\sum_{n} X_{n}\right)_{p}$ and the operator

$$
T: \ell_{\tilde{s}}(X) \longrightarrow\left(\sum_{n} X_{n}\right)_{p}, T\left(\left(w_{j}\right)_{j=1}^{\infty}\right)=\sum_{j=1}^{\infty} y_{j} \otimes w_{j},
$$

is well defined. From (6) it follows easily that $T$ is linear, and from the fact that the sequences $\left(y_{j}\right)_{j=1}^{\infty}$ are disjointly supported it follows that $T$ is injective. 
Thus $\overline{T\left(\ell_{\tilde{s}}(X)\right)}$ is a closed infinite-dimensional subspace of $\left(\sum_{n} X_{n}\right)_{p}$ and

$$
\operatorname{dim} \overline{T\left(\ell_{\tilde{s}}(X)\right)}=\operatorname{dim} \ell_{\tilde{s}}(X)=\operatorname{dim} X .
$$

Now we just have to show that

$$
\overline{T\left(\ell_{\tilde{s}}(X)\right)}-\{0\} \subseteq\left(\sum_{n} X_{n}\right)_{p}-\bigcup_{0<q<p}\left(\sum_{n} X_{n}\right)_{q} .
$$

Let $z=\left(z_{n}\right)_{n=1}^{\infty} \in \overline{T\left(\ell_{\tilde{s}}(X)\right)}, z \neq 0$. There are sequences $\left(w_{i}^{(k)}\right)_{i=1}^{\infty} \in \ell_{\tilde{s}}(X)$, $k \in \mathbb{N}$, such that $z=\lim _{k \rightarrow \infty} T\left(\left(w_{i}^{(k)}\right)_{i=1}^{\infty}\right)$ in $\left(\sum_{n} X_{n}\right)_{p}$. Note that, for each $k \in \mathbb{N}$,

$$
T\left(\left(w_{i}^{(k)}\right)_{i=1}^{\infty}\right)=\sum_{i=1}^{\infty} y_{i} \otimes w_{i}^{(k)}=\sum_{i=1}^{\infty}\left(\sum_{j=1}^{\infty} \xi_{j} e_{i_{j}}\right) \otimes w_{i}^{(k)}=\sum_{i=1}^{\infty} \sum_{j=1}^{\infty} \xi_{j} e_{i_{j}} \otimes w_{i}^{(k)},
$$

which means that, for every $i, j \in \mathbb{N}$, the $i_{j}$-th coordinate of $T\left(\left(w_{i}^{(k)}\right)_{i=1}^{\infty}\right)$ is $\xi_{j} R_{i_{j}}\left(w_{i}^{(k)}\right)$. Fix $r \in \mathbb{N}$ such that $z_{r} \neq 0$. Since $\mathbb{N}=\bigcup_{j=1}^{\infty} \mathbb{N}_{j}$, there are (unique) $m, t \in$ $\mathbb{N}$ such that $m_{t}=r$. Thus, for each $k \in \mathbb{N}$, the $r$-th coordinate of $T\left(\left(w_{i}^{(k)}\right)_{i=1}^{\infty}\right)$ is $\xi_{t} R_{r}\left(w_{m}^{(k)}\right)$. Since convergence in $\left(\sum_{n} X_{n}\right)_{p}$ implies coordinatewise convergence, we have $z_{r}=\lim _{k \rightarrow \infty} \xi_{t} R_{r}\left(w_{m}^{(k)}\right)$. From $z_{r} \neq 0$ it follows that $\xi_{t} \neq 0$ and

$$
z_{r}=\lim _{k \rightarrow \infty} \xi_{t} R_{r}\left(w_{m}^{(k)}\right)=\xi_{t} \cdot \lim _{k \rightarrow \infty} R_{r}\left(w_{m}^{(k)}\right) \in \overline{R_{r}(X)}=R_{r}(X) .
$$

Hence $R_{r}^{-1}\left(z_{r}\right)=\xi_{t} \cdot \lim _{k \rightarrow \infty} w_{m}^{(k)}$. Call

$$
\alpha_{m}:=\lim _{k \rightarrow \infty} w_{m}^{(k)}=\frac{R_{r}^{-1}\left(z_{r}\right)}{\xi_{t}} \neq 0
$$

For $j, k \in \mathbb{N}$, the $m_{j}$-th coordinate of $T\left(\left(w_{i}^{(k)}\right)_{i=1}^{\infty}\right)$ is $\xi_{j} R_{m_{j}}\left(w_{m}^{(k)}\right)$. On the one hand,

$$
\lim _{k \rightarrow \infty} \xi_{j} R_{m_{j}}\left(w_{m}^{(k)}\right)=\xi_{j} \cdot \lim _{k \rightarrow \infty} R_{m_{j}}\left(w_{m}^{(k)}\right)=\xi_{j} R_{m_{j}}\left(\lim _{k \rightarrow \infty} w_{m}^{(k)}\right)=\xi_{j} R_{m_{j}}\left(\alpha_{m}\right)
$$

for every $j \in \mathbb{N}$. On the other hand, coordinatewise convergence gives

$$
\lim _{k \rightarrow \infty} \xi_{j} R_{m_{j}}\left(w_{m}^{(k)}\right)=z_{m_{j}}
$$

so $z_{m_{j}}=\xi_{i} R_{m_{j}}\left(\alpha_{m}\right)$ for each $j \in \mathbb{N}$. Finally, for all $q$ with $0<q<p$,

$$
\begin{aligned}
\|z\|_{q}^{q} & =\sum_{n=1}^{\infty}\left\|z_{n}\right\|_{X_{n}}^{q} \geq \sum_{j=1}^{\infty}\left\|z_{m_{j}}\right\|_{X_{m_{j}}}^{q}=\sum_{j=1}^{\infty}\left|\xi_{j}\right|^{q} \cdot\left\|R_{m_{j}}\left(\alpha_{m}\right)\right\|_{X_{m_{j}}}^{q} \\
& \geq \sum_{j=1}^{\infty}\left|\xi_{j}\right|^{q} \cdot \frac{1}{\left\|R_{m_{j}}^{-1}\right\|^{q}} \cdot\left\|\alpha_{m}\right\|_{X}^{q} \geq \frac{1}{\delta^{q}} \cdot\left\|\alpha_{m}\right\|_{X}^{q} \cdot\|\xi\|_{q}^{q}=\infty,
\end{aligned}
$$

proving that $z \notin \bigcup_{0<q<p}\left(\sum_{n} X_{n}\right)_{q}$. 
(b) Start with a sequence $\xi=\left(\xi_{j}\right) \in c_{0}-\bigcup_{p>0} \ell_{p}$ and proceed as before to define the operator

$$
T: \ell_{1}(X) \longrightarrow\left(\sum_{n} X_{n}\right)_{0}, T\left(\left(w_{j}\right)_{j=1}^{\infty}\right)=\sum_{j=1}^{\infty} y_{j} \otimes w_{j} .
$$

The well-definiteness of $T$ is even easier in this case. Again $T$ is linear, injective, and the same steps of the proof of (a) show that

$$
\overline{T\left(\ell_{1}(X)\right)}-\{0\} \subseteq\left(\sum_{n} X_{n}\right)_{0}-\bigcup_{p>0}\left(\sum_{n} X_{n}\right)_{p} .
$$

As $\operatorname{dim} \overline{T\left(\ell_{1}(X)\right)}=\operatorname{dim} X$, the proof is complete.

Let $\left(X_{n}\right)_{n=1}^{\infty}$ be a sequence of Banach spaces and $1 \leq p<+\infty$. We define

$$
\left(\sum_{n} X_{n}\right)_{p}^{+}:=\bigcap_{q>p}\left(\sum_{n} X_{n}\right)_{q}=\bigcap_{k \in \mathbb{N}}\left(\sum_{n} X_{n}\right)_{p_{k}},
$$

where $\left(p_{k}\right)_{k=1}^{\infty}$ is any decreasing sequence converging to $p$, endowed with the locally convex topology $\tau$ generated by the family of norms

$$
\left\|\left(x_{n}\right)_{n=1}^{\infty}\right\|_{q}=\left(\sum_{n=1}^{\infty}\left\|x_{n}\right\|_{X_{n}}^{q}\right)^{\frac{1}{q}}, q>p .
$$

This locally convex topology $\tau$ is clearly generated by the countable family of norms

$$
\left\|\left(x_{n}\right)_{n=1}^{\infty}\right\|_{p_{k}}=\left(\sum_{n=1}^{\infty}\left\|x_{n}\right\|_{X_{n}}^{p_{k}}\right)^{\frac{1}{p_{k}}}, k \in \mathbb{N},
$$

so $\left(\left(\sum_{n} X_{n}\right)_{p}^{+}, \tau\right)$ is metrizable. The completeness can be proved similarly to the case of $\left(\sum_{n} X_{n}\right)_{p}$. Alternatively, note that $\tau$ is the projective limit topology defined by the inclusions $\left(\sum_{n} X_{n}\right)_{p}^{+} \hookrightarrow\left(\sum_{n} X_{n}\right)_{q}, q>p$. So it is complete as the projective limit of complete Hausdorff spaces. In summary $\left(\left(\sum_{n} X_{n}\right)_{p}^{+}, \tau\right)$ is a Fréchet space.

If $X_{n}=X$ for every $n$, we write $\ell_{p}(X)^{+}$. In particular, for $X=\mathbb{K}, \ell_{p}^{+}:=\ell_{p}^{+}(\mathbb{K})$ recovers the space $l^{p+}$ introduced by Metafune and Moscatelli [17.

Remark 3.3. It is easy to see that if $X_{n} \neq\{0\}$ for every $n$, then the inclusions

$$
\left(\sum_{n} X_{n}\right)_{p} \subseteq\left(\sum_{n} X_{n}\right)_{p}^{+} \subseteq\left(\sum_{n} X_{n}\right)_{q}
$$

are strict whenever $1 \leq p<q$.

Theorem 3.4. Let $\left(X_{n}\right)_{n=1}^{\infty}$ be a sequence of Banach spaces that contains a subsequence containing isomorphs of the infinite-dimensional Banach space $X$ uniformly. Then $\left(\sum_{n} X_{n}\right)_{p}^{+}-\left(\sum_{n} X_{n}\right)_{p}$ is $\operatorname{dim} X$-spaceable.

Proof. The proof goes along the same steps of the proof of Theorem 3.2. We sketch the argument highlighting the differences arising from the locally convex topology of $\left(\sum_{n} X_{n}\right)_{p}^{+}$. In this proof, as before means as in the proof of Theorem 3.2

Let $\xi=\left(\xi_{j}\right) \in \ell_{p}^{+}-\ell_{p}$. Write

$$
\mathbb{N}=\bigcup_{i=1}^{\infty} \mathbb{N}_{i}, \quad \mathbb{N}_{i}=\left\{i_{1}<i_{2}<\ldots\right\},
$$

and define $y_{j} \in \mathbb{K}^{\mathbb{N}}, j \in \mathbb{N}$, as before. Since $\left\|y_{i}\right\|_{r}=\|\xi\|_{r}$ for every $r>0$, we have that $y_{i} \in \ell_{p}^{+}-\ell_{p}$, for every $i$. Given $\left(w_{j}\right)_{j=1}^{\infty} \in \ell_{1}(X)$, let us show that the 
series $\sum_{j=1}^{\infty} y_{j} \otimes w_{j}$ converges in $\left(\sum_{n} X_{n}\right)_{p}^{+}$, where $y_{j} \otimes w_{j}$ is defined as before. Write $s_{n}=\sum_{j=1}^{n} y_{j} \otimes w_{j}, n \in \mathbb{N}$. For a fixed $q>p$, the same computations we performed before show that each $y_{j} \otimes w_{j} \in\left(\sum_{n} X_{n}\right)_{q}$ and

$$
\sum_{j=1}^{n}\left\|y_{j} \otimes w_{j}\right\|_{q} \leq \delta\|\xi\|_{q} \cdot\left\|\left(w_{j}\right)_{j=1}^{\infty}\right\|_{1}
$$

for every $n$. As $\left(\sum_{n} X_{n}\right)_{q}$ is a Banach space, there is $S_{q} \in\left(\sum_{n} X_{n}\right)_{q}$ such that $S_{q}=\lim _{n \rightarrow \infty} s_{n}$ in $\left(\sum_{n} X_{n}\right)_{q}$. If $q, q^{\prime}>p$, say $q \leq q^{\prime}$, then $S_{q} \in\left(\sum_{n} X_{n}\right)_{q^{\prime}}$ and

$$
\left\|s_{n}-S_{q}\right\|_{q^{\prime}} \leq\left\|s_{n}-S_{q}\right\|_{q} \longrightarrow 0,
$$

showing that $S_{q}=\lim _{n \rightarrow \infty} s_{n}$ in $\left(\sum_{n} X_{n}\right)_{q^{\prime}}$; therefore $S_{q}=S_{q^{\prime}}$. This shows that $S_{q}$ does not depend on $q$, so there is $S \in\left(\sum_{n} X_{n}\right)_{q}$ such that $s_{n} \longrightarrow S$ in $\left(\sum_{n} X_{n}\right)_{q}$ for every $q>p$. Hence $S \in\left(\sum_{n} X_{n}\right)_{q}^{+}$and $s_{n} \longrightarrow S$ in the topology of $\left(\sum_{n} X_{n}\right)_{p}^{+}$. In other words, $\sum_{j=1}^{\infty} y_{j} \otimes w_{j} \in\left(\sum_{n} X_{n}\right)_{p}^{+}$and the operator

$$
T: \ell_{1}(X) \longrightarrow\left(\sum_{n} X_{n}\right)_{p}^{+}, T\left(\left(w_{j}\right)_{j=1}^{\infty}\right)=\sum_{j=1}^{\infty} y_{j} \otimes w_{j}
$$

is then well defined. As before, $T$ is linear and injective. Thus $\overline{T\left(\ell_{1}(X)\right)}$ is a closed $\operatorname{dim} X$-dimensional subspace of $\left(\sum_{n} X_{n}\right)_{p}^{+}$. Now we just have to show that

$$
\overline{T\left(\ell_{1}(X)\right)}-\{0\} \subseteq\left(\sum_{n} X_{n}\right)_{p}^{+}-\left(\sum_{n} X_{n}\right)_{p} .
$$

Let $z=\left(z_{n}\right)_{n=1}^{\infty} \in \overline{T\left(\ell_{1}(X)\right)}, z \neq 0$. There are sequences $w_{k}=\left(w_{i}^{(k)}\right)_{i=1}^{\infty} \in \ell_{1}(X)$, $k \in \mathbb{N}$, such that $z=\lim _{k \rightarrow \infty} T\left(\left(w_{i}^{(k)}\right)_{i=1}^{\infty}\right)$ in $\left(\sum_{n} X_{n}\right)_{p}^{+}$. Since the topology of $\left(\sum_{n} X_{n}\right)_{p}^{+}$is generated by the norms $\|\cdot\|_{q}, q>p$, it follows that

$$
\lim _{k \rightarrow \infty}\left\|T\left(\left(w_{i}^{(k)}\right)_{i=1}^{\infty}\right)-z\right\|_{q}=0 \text { for every } q>p .
$$

Fix $q>p$ and use coordinatewise convergence in $\left(\sum_{n} X_{n}\right)_{q}$ as before to conclude that $z \notin\left(\sum_{n} X_{n}\right)_{p}$.

Making $X_{n}=X$ for every $n$ in Theorems 3.2 and 3.4. since $\operatorname{dim} \ell_{p}(X)=$ $\operatorname{dim} c_{0}(X)=\operatorname{dim} \ell_{p}(X)^{+}=\operatorname{dim} X$, we get:

Corollary 3.5. Let $X$ be an infinite-dimensional Banach space $X$. Then:

(a) $\ell_{p}(X)-\ell_{p}(X)^{-}$is maximal-spaceable for every $0<p<\infty$.

(b) $c_{0}(X)-\bigcup_{p>0} \ell_{p}(X)$ is maximal-spaceable.

(c) $\ell_{p}(X)^{+}-\ell_{p}(X)$ is maximal-spaceable for every $1 \leq p<\infty$.

Of course Theorems 3.2 and 3.4 apply to many other interesting situations, but we refrain from going into details.

Remark 3.6. Note that in the proofs of Theorems 3.2 and 3.4 we start with a vector $\xi$ belonging to a set other than the one we prove to be spaceable. It is this variation of the mother vector technique that allows us to obtain maximal-spaceability rather than spaceability in Corollary 3.5 


\section{ACKNOWLEDGEMENTS}

This work was started when the first author was visiting the Department of Mathematics of the Universidade Federal da Paraiba in 2010; he thanks the members of the Department of Mathematics for their kind hospitality. The authors thank J. M. Ansemil for drawing their attention to the spaces $\ell_{p}^{+}$and for pointing out reference [17. The authors also thank the referee for important suggestions and corrections that improved the final version of this paper.

\section{REFERENCES}

[1] R. M. Aron, F. J. García-Pacheco, D. Pérez-García and J. B. Seoane-Sepúlveda, On denselineability of sets of functions on $\mathbb{R}$, Topology 48 (2009), 149-156. MR2596209|(2011c:26011)

[2] R. M. Aron, V. I. Gurariy and J. B. Seoane-Sepúlveda, Lineability and spaceability of sets of functions on $\mathbb{R}$, Proc. Amer. Math. Soc. 133 (2005), 795-803. MR2113929 (2006i:26004)

[3] K. Astala, On Peano's theorem in locally convex spaces, Studia Math. 73 (1982), 213-223. MR675425(84m:34090)

[4] G. Botelho, D. Diniz, V. Fávaro and D. Pellegrino, Spaceability in Banach and quasi-Banach sequence spaces, Linear Algebra Appl. 434 (2011), 1255-1260. MR2763584

[5] A. Cellina, On the nonexistence of solutions of differential equations in nonreflexive spaces, Bull. Amer. Math. Soc. 78 (1972), 1069-1072. MR0312017 (47:579)

[6] J. Dieudonné, Deux exemples singuliers d'équations différentielles, Acta Sci. Math. (Szeged) 12B (1950), 38-40. MR0035397(11:729d)

[7] J. L. Gámez-Merino, G. A. Muñoz-Fernández, V. M. Sánchez and J. B. Seoane-Sepúlveda, Sierpiński-Zygmund functions and other problems on lineability, Proc. Amer. Math. Soc. 138 (2010), no. 11, 3863-3876. MR2679609 (2011g:46042)

[8] F. J. García-Pacheco and J. B. Seoane-Sepúlveda, Vector spaces of non-measurable functions, Acta Math. Sin. (Engl. Ser.) 22 (2006), 1805-1808. MR2262440 (2007i:28006)

[9] A. N. Godunov, Counterexample to Peano's theorem in infinite-dimensional Hilbert space, Vestnik Mosko. Univ. Ser. 1 Mat. Mekh. [Moscow Univ. Math. Bull.] 5 (1972), 19-21.

[10] A. N. Godunov, On Peano's theorem in Banach spaces, Funktsional. Anal. i Prilozhen. [Functional Anal. Appl.] 9 (1975), 59-60. MR0364797 (51:1051)

[11] P. Hájek and M. Johanis, On Peano's theorem in Banach spaces, J. Differential Equations 249 (2010), 3342-3351. MR2737433 (2011j:34182)

[12] D. Kitson and R. M. Timoney, Operator ranges and spaceability, J. Math. Anal. Appl. 378 (2011), 680-686. MR2773276

[13] A. Lasota and J. A. Yorke, The generic property of existence of solutions of differential equations in Banach spaces, J. Differential Equations 13 (1973), 1-12. MR0335994(49:770)

[14] S. G. Lobanov, Peano's theorem is invalid for any infinite-dimensional Fréchet space (Russian), Mat. Sb. 184 (1993), 83-86; translation in Russian Acad. Sci. Sb. Math. 78 (1994), 211-214. MR 1214945(93m:34095)

[15] S. G. Lobanov and O. G. Smolyanov, Ordinary differential equations in locally convex spaces (Russian), Uspekhi Mat. Nauk. 49 (1994), 93-168; translation in Russian Math. Surveys 49 (1994), 97-175. MR1289388 (95k:34002)

[16] J. López-Salazar, Vector spaces of entire functions of unbounded type, Proc. Amer. Math. Soc. 139 (2011), 1347-1360. MR2748427

[17] G. Metafune and V. B. Moscatelli, On the space $l^{p+}=\bigcap_{q>p} l^{q}$, Math. Nachr. 147 (1990), 7-12. MR $1127304(92 \mathrm{i}: 46005)$

[18] S. A. Shkarin, Peano's theorem in infinite-dimensional Fréchet spaces is invalid (Russian), Funktsional. Anal. i Prilozhen. 27 (1993), 90-92; translation in Funct. Anal. Appl. 27 (1993), 149-151. MR 1251174 (94h:34080)

[19] S. A. Shkarin, Peano's theorem is invalid in infinite-dimensional $F^{\prime}$-spaces (Russian), Mat. Zametki 62 (1997), 128-137; translation in Math. Notes 62 (1997), 108-115. MR.1620008 (99c:34140)

[20] J. A. Yorke, A continuous differential equation in Hilbert space without existence, Funkcial. Ekvac. 13 (1970), 19-21. MR0264196(41:8792) 
Departamento de Matemática, Campus do Pici, Universidade Federal do Ceará, 60.455-760 Fortaleza, Brazil

E-mail address: cleonbar@mat.ufc.br

Faculdade de Matemática, Universidade Federal de Uberlândia, 38.400-902, UBERLÂNDIA, BRAZIL

E-mail address: botelho@ufu.br

Faculdade de Matemática, Universidade Federal de Uberlândia, 38.400-902, UBERLÂNDIA, BRAZIL

E-mail address: vvfavaro@gmail.com

Departamento de Matemática, Universidade Federal da Paraíba, 58.051-900, João PESSOA, BRAZIL

E-mail address: pellegrino@pq.cnpq.br

E-mail address: dmpellegrino@gmail.com 